\title{
Thermal Behavior of Glassy Phase Stabilized Ammonium Nitrate (PSAN) Thin Films
}

J. D. Yeager ${ }^{1, *}$, R. Chellappa ${ }^{2}$, S. Singh ${ }^{2}$, J. Majewski ${ }^{2}$

${ }^{1}$ Shock and Detonation Physics, Los Alamos National Laboratory, Los Alamos, NM 87545, USA

${ }^{2}$ Lujan Neutron Scattering Center, Los Alamos National Laboratory, Los Alamos, NM 87545, USA

*Corresponding Author: jyeager@lanl.gov, +001 505- －0879. PO Box 1663, MS P952, Los Alamos, NM 87545, USA

\begin{abstract}
Ammonium nitrate $(\mathrm{AN})$ is a high interest material because of its wide usage in propellants and explosives but can be difficult to handle from a formulation standpoint. It is soluble in many common solvents and has complex phase behavior. Here, we formulate phase stabilized AN (PSAN) films in a polymer matrix and characterize thermal and phase behavior using neutron reflectometry and ellipsometry. These techniques are highly sensitive to small changes in composition and density which occur during phase transitions. Our PSAN films are generally stable up to $160^{\circ} \mathrm{C}$, though we observe small material loss between 60 and $100^{\circ} \mathrm{C}$, which we attribute to solvent interactions with the PSAN. Crystallization of AN from supersaturated polymer is most common at thicker regions of the film, suggesting a critical nucleation thickness for the AN which can be avoided by making very thin films.
\end{abstract}

Keywords: ammonium nitrate; neutron reflectometry; phase transformation 


\section{Introduction}

Ammonium Nitrate $\left(\mathrm{AN}\right.$ : $\left.\mathrm{NH}_{4} \mathrm{NO}_{3}\right)$ is an important chemical that is widely used as a fertilizer component as well as for energetic applications [1, 2]. Polymorphism in AN has been extensively investigated with numerous phase transitions as a function of pressure and temperature [3]. At ambient pressure, in the temperature regime relevant to this work, there are three phases (dry AN) denoted as $\operatorname{AN}-I V\left(25-51^{\circ} \mathrm{C}\right)$, AN-II $\left(51-126^{\circ} \mathrm{C}\right)$, AN-I $\left(126-169^{\circ} \mathrm{C}\right)$, and a fourth intermediate $\mathrm{AN}-\mathrm{III}\left(32-84{ }^{\circ} \mathrm{C}\right)$ phase that arises under certain conditions (including higher moisture content). The IV $\rightarrow$ III phase transition which occurs close to ambient temperature is an isomorphous order-disorder transformation between two orthorhombic structures, Pmmn (IV) and Pnma (III). This transformation is characterized by a large volume change and the crystallographic axes are not preserved resulting in fracturing, loss of crushing strength, and overall degradation to a powdered form. This has significant implications for storage and handling (especially large quantities in fertilizer warehouses) as well as for performance in energetic applications. Therefore, a great deal of research has been devoted to creating the so-called phase stabilized AN (PSAN) by mixing AN with additives from metal powder, metal oxides, carbonates, and related nitrates [4-7]. Recently, polymeric binders for stabilizing AN (suppression of AN phase transitions) were proposed with the objective of immobilizing ammonium $\mathrm{NH}_{4}{ }^{+}$and nitrate $\mathrm{NO}_{3}^{-}$ions within the polymeric matrix [8-10]. The chosen polymer should interact with ionic moieties (i.e., high dipole moment from a strong polar functional group) with a glass transition temperature $\left(T_{\mathrm{g}}\right)$ that is sufficiently high and potential candidates include poly (vinyl pyrrolidone):PVP and poly (acryl amide):PAM. PVP is known to form complexes with inorganic salts (e.g. $\left.\mathrm{Li}^{+} \mathrm{ClO}_{4}^{-}\right) \mathrm{Li}+$ and $\mathrm{ClO}_{4}^{-}$associate with ' $\mathrm{C}=\mathrm{O}$ ' and ' $N$ ' centers of the pyrrolidone ring [11]. In a series of studies, Lang and Vyazovkin [8-10] 
investigated AN-PVP mixtures with varying AN content $(5,10,20 \ldots 50 \%$ AN) using thermal analysis (TG-DSC) and vibrational spectroscopy. Remarkably, thick films $(0.5 \mathrm{~mm})$ of AN-PVP $(20,25 \%$ AN) revealed that the three solid-solid transitions $(\mathrm{IV} \rightarrow \mathrm{III}, \mathrm{III} \rightarrow \mathrm{II}, \mathrm{II} \rightarrow \mathrm{I})$ were fully suppressed and a single exotherm at $\sim 160{ }^{\circ} \mathrm{C}$ close to the Tg of PVP was observed by DSC. The mass loss associated with this thermal event is close to the amount of AN indicating that breakdown of the PVP matrix results in decomposition of AN. FTIR spectra showed that the binding of AN to PVP is primarily due to the ion-dipole interaction between the $\mathrm{C}=\mathrm{O}$ and $\mathrm{NH}_{4}{ }^{+}$ ions and confirmed using DFT calculations.

Surface modifications of AN due to ambient moisture, temperature fluctuations, stress and other factors can lead to deliquescence, grain coagulation, and nucleation of undesirable phase transformations. This can result in severe safety issues during bulk fertilizer storage as well as impacting performance in energetic applications. Under rapid heating, decomposition behavior of surface (or thin films) is affected by these modifications, resulting in low surface temperature and slow burn rate. The mechanisms of surface reactions are expected to be more complicated than bulk. Optical, thermal, and spectroscopic studies have shown that individual grains of AN can coagulate, resulting in a complicated thermal response and phase transformations occurring across a temperature gradient dependent upon particle size $[12,13]$. Research has focused on using various organic [14] and mineral [15] coatings to avoid some of these surface phenomena however this conditioning can result in deterioration of explosive performance $[16,17]$.

Here, we attempt to investigate the stability of AN in a PVP matrix by detailed characterization of composite films. The primary goal is to observe phase stabilization and / or structural effects such as material loss (presumably AN decomposition) during heating experiments. We focus on 
the nanoscale in order to study surfaces and interfaces (e.g. AN-substrate interactions) because our previous research has indicated that precipitation of crystalline material from binders can happen on the nanoscale and such precipitates may have scaled effects not observable with bulk techniques. NR experiments provide precise composition and density measurements throughthickness, and here are performed at several temperatures in between known phase transition temperatures for $\mathrm{AN}$ as well as near the glass transition temperature for PVP. Ellipsometry is used to provide experimental parameters for more realistic modeling of the reflectometry data, while polarized light microscopy is used to identify crystallization of AN from the PVP and give a microscopic overview of the thin film samples. Finally, we use DSC-TGA to complement the mass-loss and phase behavior information given by thermal reflectometry measurements.

\section{Materials and Methods}

\subsection{Preparation of samples}

Most of the techniques used here require thin films on the order of $1-500 \mathrm{~nm}$. Generally speaking, films of such a size are unstable as standalone materials, so the films were prepared on silicon substrates (Silicon Sense, Inc., Neshua, NH). The substrates were cleaned prior to film deposition using sequential washing in acetone, methanol, and deionized water. Ammonium nitrate (99\% Purity, Sigma Aldrich) and poly(vinylpyrrolidone) PVP K30 (ISP Technologies Inc., Wayne, NJ) were mixed together in three ratios $(10 \mathrm{wt} \% \mathrm{AN}, 25 \mathrm{wt} \% \mathrm{AN}$, and $40 \mathrm{wt} \% \mathrm{AN}$ in PVP) and dissolved in methanol to form a dilute solution (1-2 wt\% total solids) that is amenable for creating films of desired thickness. It is noted that the choice of AN-PVP ratios is guided by the earlier work which report homogeneous, stable solid solution in PVP up to $25 \mathrm{wt} \%$ AN and unstable (AN precipitates formed) above $25 \mathrm{wt} \%$ [8]. 
Films were prepared by dipping the silicon into the methanol solution and slowly removing in a glove-box, as in [18]. Figure 1 shows a macroscopic view of the resulting sample. If environmental conditions such as air currents, humidity and temperature are controlled appropriately, the deposited film is generally uniform with regions of greater thickness at the very top of the film (caused by interactions with the surface of the solution) and at the edges. However, if the substrate is large enough, the uniform area is correspondingly large enough for NR measurements, which generally require square centimeters of surface area for statistically significant data. It is noted that bulk samples for thermal analysis were prepared separately by simply evaporating the solvent in a roto-evaporator and the sample was isolated from ambient.

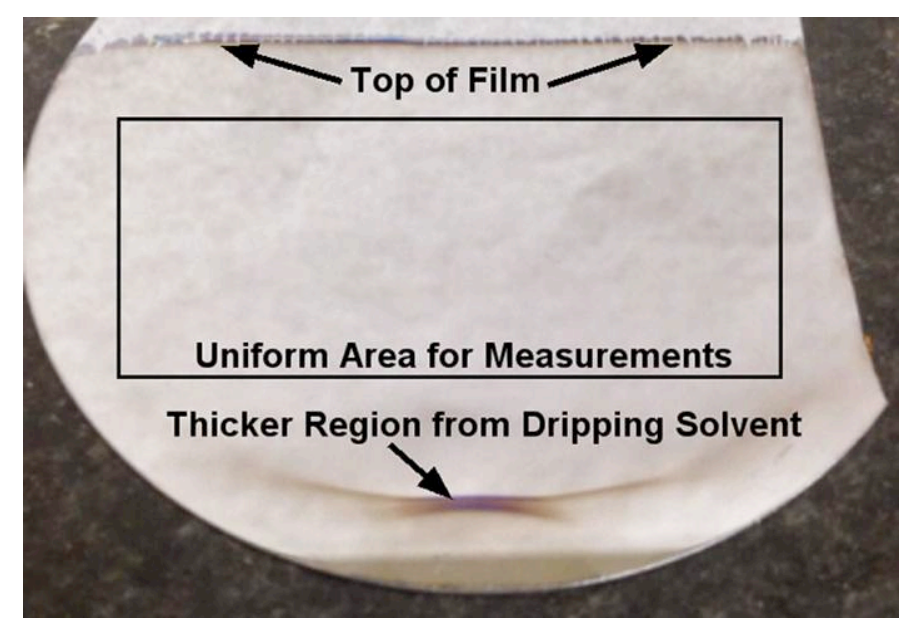

Figure 1. Photograph of a silicon substrate with an AN-PVP film deposited via dip-coating. The width of the uniform area is approximately $40 \mathrm{~mm}$.

\subsection{Preliminary Sample Characterization}

Samples were characterized in all cases with polarized light microscopy (PLM) after 48 hours. This was judged to be a sufficient time for the solvent to evaporate and the films to stabilize. 
Polarized light allows for enhanced differentiation of crystalline regions from amorphous regions [19-21]. PLM was also performed after two months to assess the stability of the AN within the film. Two sets of samples were examined and compared: 1) the thermally treated samples measured by NR, and 2) a separate set of samples which were not thermally treated.

Simultaneous differential scanning calorimetry and thermogravimetric analysis (DSC-TGA) was performed on bulk solid samples to confirm earlier reports of composition dependent suppression of solid-state phase transitions of AN as well as increased thermal stability [8]. A moderate heating rate of $5^{\circ} \mathrm{C} / \mathrm{min}$ was adopted for ramping temperature from $25^{\circ} \mathrm{C}$ to $325^{\circ} \mathrm{C}$. It is noted that this heating profile is significantly accelerated compared to a typical NR measurement, as discussed below. However, the DSC-TGA scans provide a baseline for expected (or suppressed) thermal events to interpret NR measurements (see Section 3.2).

\subsection{Ellipsometry and Neutron Reflectometry}

We have previously demonstrated the usefulness of combining ellipsometry and NR to provide through-thickness compositional information of complex films [18, 22, 23]. Those works describe in detail the parameters for such experiments and so we will not include the details here. Briefly, both techniques use reflected particles (photons and neutrons) with highly characterized input properties and measure the changes in those properties after reflecting off of the sample. Figure 2 illustrates these techniques schematically. 


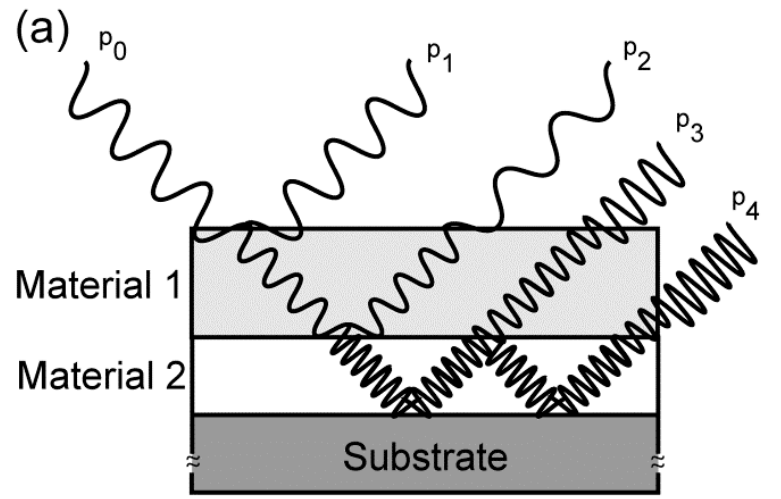

Ellipsometry (b)

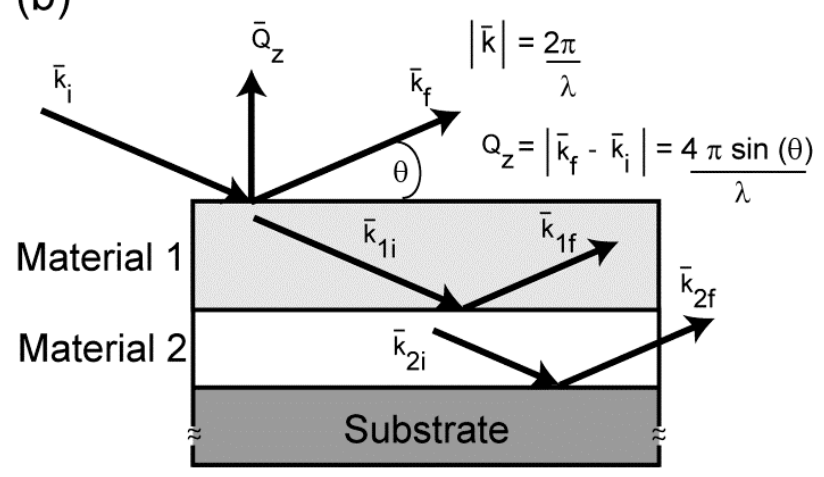

Neutron Reflectometry

Figure 2. Schematic descriptions of ellipsometry (a) and neutron reflectometry (b), showing similarities and differences between the techniques. The change in polarization $(p)$ is measured with ellipsometry, while reflectometry measures the change in neutron intensity as a function of neutron momentum transfer $\left(Q_{z}\right) . Q_{z}$ is a function of neutron wavelength $(\lambda)$ and angle of reflection $(\theta)$.

Ellipsometry uses polarized light with a known polarization $(p)$, amplitude $(\Psi)$ and phase $(\Delta)$, such that $p=\tan (\Psi) e^{i \Delta}$. If the complex refractive index (refractive index $n$ and absorption $\kappa$ ) and thickness of the film are both unknown initially, particularly for samples which are heterogeneous, a unique solution for both parameters requires measuring the sample at multiple angles of incidence over a range of light frequencies. For these experiments, a variable angle spectroscopic ellipsometer (VASE, J.A. Woolam Co., Inc, Lincoln, NE) was used measure the polarization of the reflected light at discrete input frequencies (spaced every $100 \AA$ ) within the wavelength range $4000-10000 \AA$. Each sample was measured in multiple locations at 65, 70 and $75^{\circ}$ angles of incidence. Model fits of the data were generated using a stacked layer approach, wherein each layer was modeled as either a homogeneous composition or a regular 
heterogeneous mixture. The VASE software accomplishes the latter by using an effective medium approximation (EMA) layer. Such a layer assumes the composition is that of two or more homogeneous materials perfectly mixed. In our samples, a variety of similar modeling approaches were attempted.

NR was performed at the Surface Profile Analysis Reflectometer (SPEAR) at the Lujan Neutron Scattering Center in Los Alamos, NM [24]. For these experiments, the samples were measured in a custom pressure-temperature cell [25] under flowing argon gas. Since NR is a time-intensive technique, usually requiring at least 30 minutes per measurement, dynamic measurements during heating are not possible. Thus in order to investigate phase stability in the AN-PVP films, we chose to hold the cell at certain temperatures in between known phase transition points in pure AN. The films were measured at ambient $\left(20^{\circ} \mathrm{C}\right), 60^{\circ} \mathrm{C}, 100^{\circ} \mathrm{C}, 140^{\circ} \mathrm{C}, 180^{\circ} \mathrm{C}$, and then once more at ambient after the films had cooled down.

NR at SPEAR is a time-of-flight technique, meaning that the wavelengths of the scattered neutrons are determined by precisely measuring the time between neutron production and detector sensing. Since the distance between the source and the detector is known, the velocity (thus energy and wavelength) is given by a simple calculation. The instrument measures reflectivity $(R)$, which is the ratio of reflected to incident beam intensity, as a function of momentum transfer normal to the sample surface $\left(Q_{z}\right)$, itself a function of $\lambda$ and $\theta$ as shown in Figure 2(b). Similarly to ellipsometry, there is no unique solution for the complex reflection in terms of the neutron scattering properties of the material and the thickness of the film. The description of the sample is given by comparing the $R$ vs. $Q_{z}$ data to a model prediction of 
scattering from an idealized sample consisting of several layers or boxes of homogeneous material. The neutron scattering itself is determined by the thickness of the various layers and their scattering length density (SLD), which is a function of neutron scattering cross section (i.e. composition) and atomic volume (i.e. density).

Detailed descriptions of the analysis procedure for both techniques are given elsewhere [26, 27]. Here, suffice it to say that both techniques use software which constructs theoretical samples and projects experimental data to compare with the measurements. Iterative model fitting then proceeds, generally by attempting to reduce differences between theoretical and experimental values. For ellipsometry, this is accomplished simply by using mean square error (MSE), while

the NR software uses genetic algorithms and least-squares regression analysis $\left(\chi^{2}\right)$. Generally, the theoretically-constructed model which has the smallest error and reasonable values of fitting parameters is considered the most accurate. However, increasing the number of layers in the model allows the fitting algorithms to better minimize the error between the model and the actual data, meaning that the quality of the model description can be artificially improved simply by adding more and more layers (whether they are meaningful or not). To avoid this, in all cases we limited the number of layers to the minimum necessary to describe the data. Specific descriptions of the data fitting will be given in the appropriate subsection within the Results, below.

\section{Results and Discussion}

\subsection{PLM and Ellipsometry}

All films were dip-coated successfully on the silicon substrates, characterized with PLM, and then measured with ellipsometry after 48 hours. This time delay allowed for observation of 
crystallization of the AN, if it happened immediately, which in our previous research on similar materials was observed to happen shortly after the residual solvent in the film evaporated [20]. PLM evaluated the time-to-crystallization of the samples, spanning from 48 hours after dipcoating to 2 months after coating. Selected micrographs are shown in Figure 3 . The 10 and 25 wt $\%$ AN-PVP films never showed crystallization within the "uniform area" from Figure 1, while the $40 \%$ film had almost no initial crystallization but a small amount after 2 months. All samples had evidence of crystallization within the thicker, non-uniform regions of the film. Examples of such regions are at the top edge of the film, along the sides of the silicon wafer, and at the bottom of the film. The conclusions of the PLM study were: 1) the 10 and $25 \mathrm{wt} \%$ AN samples were stable over several months, 2) the $40 \mathrm{wt} \%$ AN sample was not nearly as unstable as expected from the results of Lang and Vyazovkin, and 3) that crystallization apparently needed some critical thickness (in actuality, probably a critical volume) or heterogeneity (e.g. edge of sample) to nucleate and grow. The last two factors are interrelated since the film is thicker along the heterogeneities from the dip-coating process. 


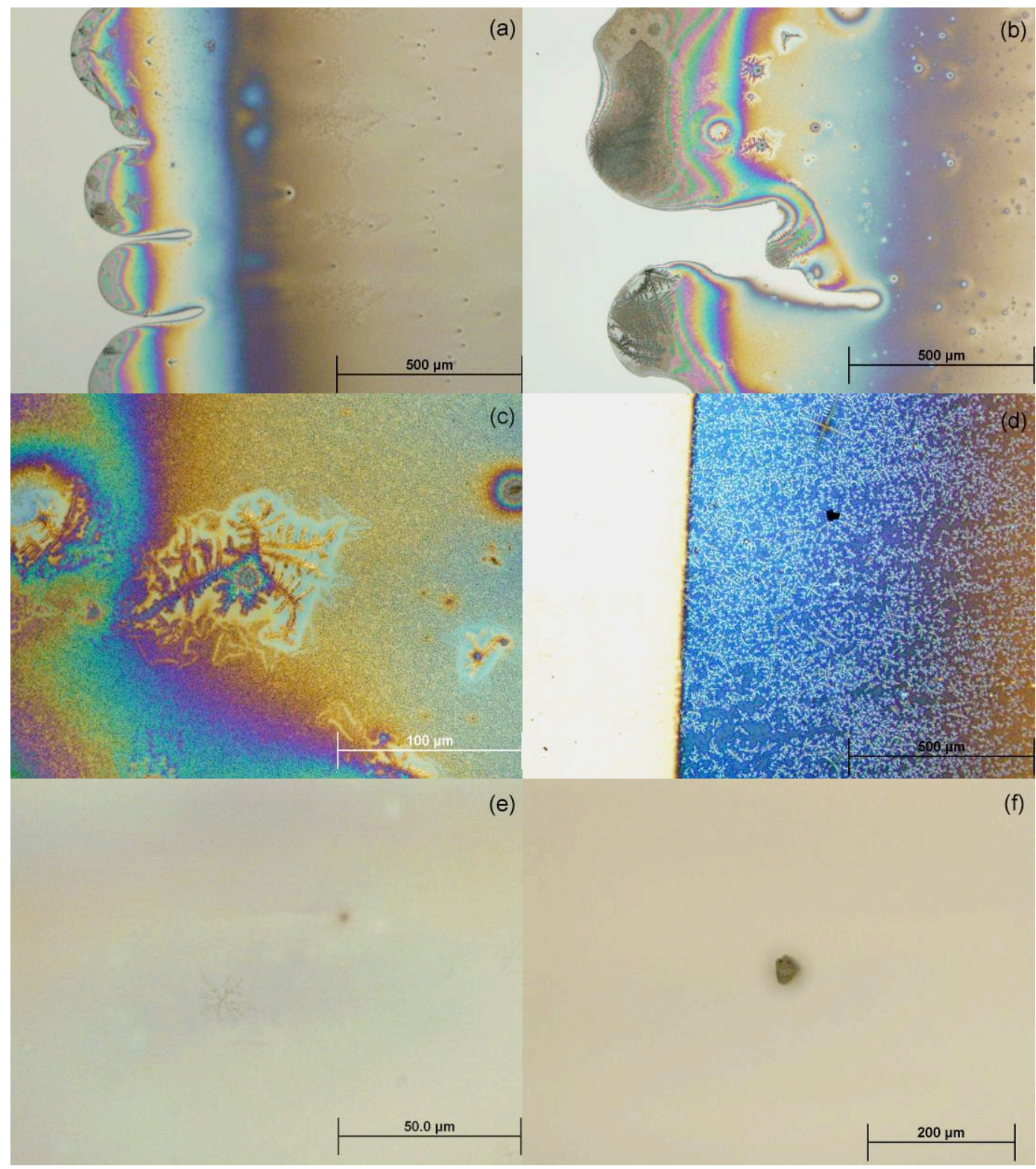

Figure 3. Polarized light micrographs of the AN-PVP samples. Crystallization of AN is seen to evolve at the edge of the $40 \mathrm{wt} \%$ film from the time of deposition (a) over a period of two months (b). A magnified view of one of the AN crystallites is shown in (c). The thickest part of the film had extensive crystallization (d) but the thin, uniform section of the film actually measured by NR had very few crystallites, few and far between (e). The $25 \mathrm{wt} \%$ film did not show any evidence of crystallization, as in (f) with a piece of dust on the surface for contrast. 
Ellipsometry was performed on multiple locations within the uniform area of the films. For the initial attempts at modeling the data, we assumed that the solid-solution AN could affect the PVP complex index of refraction. It was unknown whether the films were effectively homogeneous or heterogeneous. Three modeling approaches were thus taken, two of which used a single-layer model and one of which used a mixed layer model. These layers could then be stacked as necessary to reproduce the data. The raw data and model fits for the $10 \mathrm{wt} \%$ and $40 \mathrm{wt} \%$ samples are shown in Figure 4. The $25 \mathrm{wt} \%$ sample is not shown because it is nearly identical to the 10 wt $\%$ sample. Figure $4 \mathrm{a}$ and $4 \mathrm{c}$ show the amplitude and phase polarization data for $10 \%$ and $40 \%$ AN-PVP, respectively, while Figure $4 \mathrm{~b}$ and $4 \mathrm{~d}$ show the respective model fits to only the phase data and over a smaller range of wavelengths for clarity.

None of the three samples were fit perfectly by any single layer model (all used the same Si and $\mathrm{SiO}_{2}$ base substrates). The $10 \%$ and $25 \%$ samples were most successfully modeled by the same optical constants as a pure PVP film ("PVP Standard"). The EMA assumed a mixture of PVP and an unknown material, where $n$ and $\kappa$ of the unknown material and the ratios of the two materials could be adjusted iteratively by the software. Modeling the films as two distinct layers of PVP and EMA was also attempted. As Figure $4 \mathrm{~b}$ shows, none of the approaches were more successful than just using the PVP Standard optical parameters. In essence, the AN was not affecting the optical properties of the PVP film at 10 or $25 \mathrm{wt} \%$.

However, the $40 \mathrm{wt} \%$ film could not be modeled by the simple approaches. The best fit for the film used a two-layer structure. The layer on top was an EMA layer containing PVP and 5 atomic\% "solid crystal", while the bottom layer was a Cauchy-based EMA layer containing 35 
atomic\% solid crystal. Modeling polymer films using Cauchy dispersion models is discussed elsewhere [28] and the details are not important for this study, but generally speaking the Cauchy layer here was similar but not quite equal to the PVP Standard, itself obtained using Cauchy models . Likewise, the solid crystal was not identical to anything in literature but was used as a generic stand-in for undissolved precipitates in the film. More so than any specific identification of the phase behavior in the $40 \%$ film, the conclusion from the ellipsometry is that the structure of the film is not simple and contains both solid-solution and crystalline AN. This generally agrees with the PLM. 

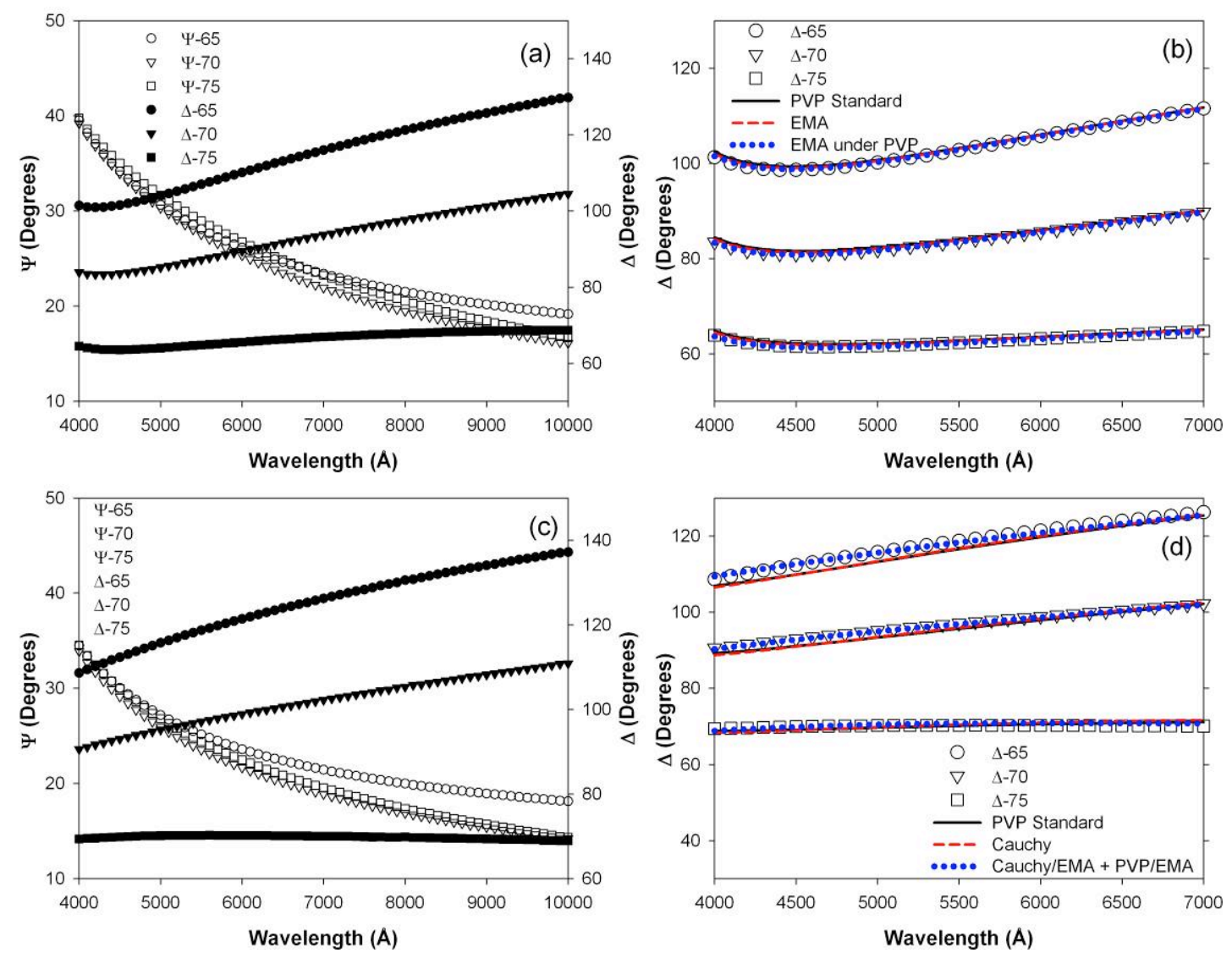

Figure 4. Ellipsometry of the $10 \mathrm{wt} \%$ AN-PVP film at 3 angles and spanning $4000-10000 \AA$ (a). Application of various model fits to the $10 \mathrm{wt} \%$ AN-PVP ellipsometry data are shown in (b). Only the shift in phase is plotted for clarity. No significant differences are observable between fitting the film as homogeneous PVP vs. fitting as a modified polymer layer. However, application of various model fits to the raw $40 \mathrm{wt} \%$ AN-PVP ellipsometry data (c) are shown in (d). Here, we see definite differences between modeling a homogeneous PVP layer vs. modeling a multi-layer film. 
The other useful result from the ellipsometry study is the final thickness of the films, shown in Table 1. This data provides a starting point for the neutron reflectometry data fitting. The optical constants listed in Table 1 are the average for the best fit of the data.

Table 1. Final film parameters from model fitting of ellipsometry data. Thickness is reported with a standard deviation.

\begin{tabular}{|l|l|l|l|}
\hline Sample & $\boldsymbol{n}$ & $\boldsymbol{k}$ & Thickness $(\mathbf{\AA})$ \\
\hline $10 \%$ AN in PVP & 1.513 & 0.004 & $565 \pm 35$ \\
\hline $25 \%$ AN in PVP & 1.513 & 0.004 & $460 \pm 17$ \\
\hline $40 \%$ AN in PVP & 1.660 & 0.117 & $406 \pm 63$ \\
\hline
\end{tabular}

\subsection{DSC-TGA}

DSC-TGA scans of bulk samples of AN-PVP mixtures (10\%, 25\%, and 40\% AN) are shown in Figure 5. The glass transition for pure PVP can vary from $101-177^{\circ} \mathrm{C}$ depending upon the molecular weight and for PVP K30 $(50,000 \mathrm{~g} / \mathrm{mol})$, the nominal value is $156^{\circ} \mathrm{C}$. As noted earlier, solid-solid phase transitions in neat AN can be complicated due to several factors and typically exhibits three endotherms at $\sim 32^{\circ} \mathrm{C}(\mathrm{IV} \rightarrow \mathrm{III}), 84^{\circ} \mathrm{C}(\mathrm{III} \rightarrow \mathrm{II})$, and $126^{\circ} \mathrm{C}(\mathrm{II} \rightarrow \mathrm{I})$ before melting (see Figure 5a). From the DSC scans in Figure 5b, the 25\% AN sample does not exhibit any of these features, and with increase in AN content to $40 \%$ AN, a small feature corresponding to $\mathrm{II} \rightarrow \mathrm{I}$ transition $\left(\sim 125^{\circ} \mathrm{C}\right)$ is observed. All three samples exhibit a strong exotherm in the vicinity of the PVP glass transition temperature. However, in the $40 \%$ AN sample, an additional peak at $190^{\circ} \mathrm{C}$ is observed, likely due to simultaneous decomposition of AN. Interestingly, the drop in mass around the glass transition temperature is almost equal to the amount of $\mathrm{AN}$ in the sample 
and a small additional loss due to release of water. From the above, we can conclude that at 10 wt.\% AN and 25 wt.\% AN, the AN-PVP mixture is in a homogeneous glassy state (glassy appearance visually) while 40 wt.\% AN contains some crystalline AN phase which exhibits features corresponding to solid-solid phase transition.

DFT calculations have suggested a possible mechanism for the suppression of AN phase transitions in excess PVP that is attributed to a separation and preferential binding of the $\mathrm{NH}^{+}$ and $\mathrm{NO}^{3-}$ ionic species to various centers of the PVP monomer. The $\mathrm{NH}_{4}^{+}$moves to interact with electron-rich ' $\mathrm{O}$ ' atom of the carbonyl group while $\mathrm{NO}^{3-}$ exhibits a weak hydrogen bonding with ' $\mathrm{H}$ ' atoms of the pyrrolidone ring. The dramatic weight loss associated with $\mathrm{AN}$ at the glass transition can be attributed due to a loss of rigidity of the PVP matrix and increasing mobility which disrupts this electrostatic interaction.

Figure 5 compares the three types of samples, plotting the results of the TGA in Figure $5 \mathrm{a}$ and of DSC in Figure 5b. Recall that for these measurements, "bulk" samples were required instead of nm-scale thin films, so the results may not replicate the exact conditions of the film samples under heating. For each sample, approximately the entire mass of AN evaporated at $\sim 170^{\circ} \mathrm{C}$, which is near the melting point of pure AN. M 

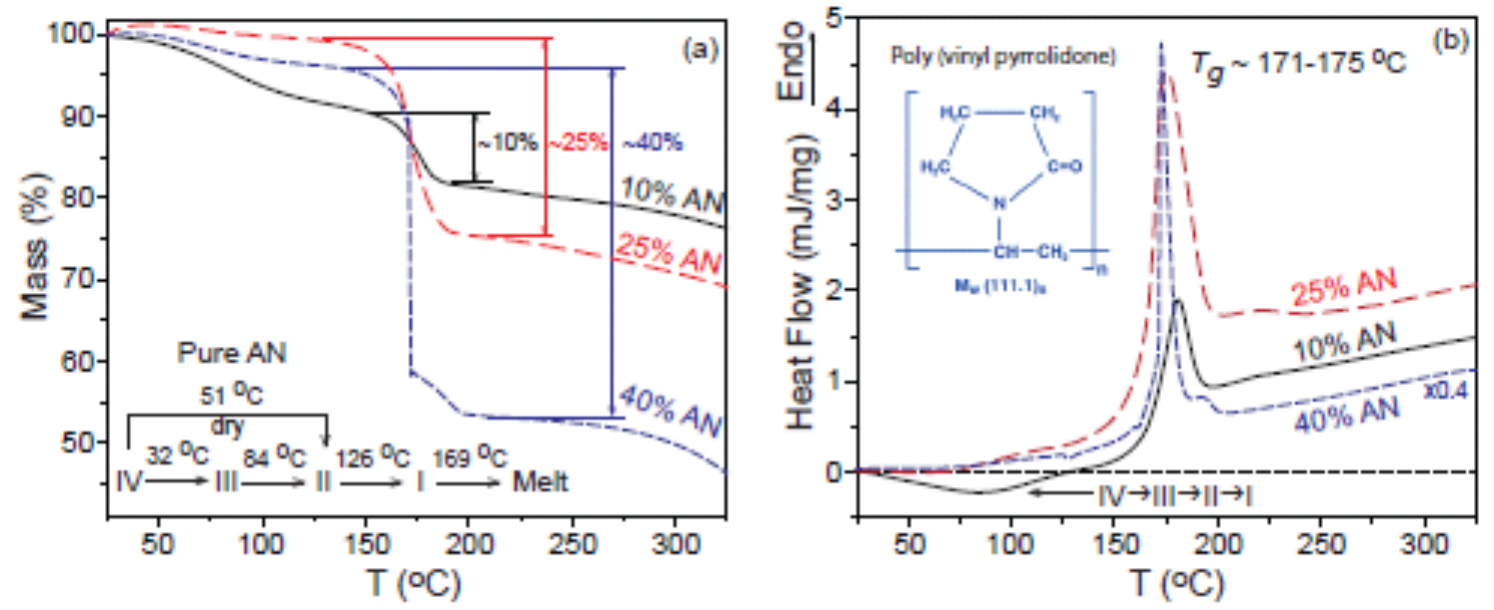

Figure 5. TGA (a) and DSC (b) results for the various AN-PVP mixtures. The TGA results are as expected, where the appropriate mass of $\mathrm{AN}$ is lost at or near the known melting temperature of the AN (molten AN decomposes and / or evaporates quickly). The amount of AN also has an increasingly dramatic effect on the $\mathrm{T}_{\mathrm{g}}$ of the PVP film, seen in the DSC data.

\section{$\underline{3.3 \text { Neutron Reflectometry }}$}

As mentioned in Section 2, NR was conducted at SPEAR by heating the samples in an inert atmosphere and holding at various temperatures for approximately 30 minutes. Typical NR experimental data, along with a model fit, are shown in Figure 6. Characteristically, the highest reflectivity $R$ is seen with the lowest $Q_{z}$ neutron momentum transfers Below the critical momentum transfer value the reflectivity is found to be equal to one, and thus $\log (R)=0$. The critical edge and the nature of the "fringes" or peaks and valleys in the data are primarily the features that the software tries to fit. Spacing between the fringes and their heights are determined by the SLD and the thickness of the film. 

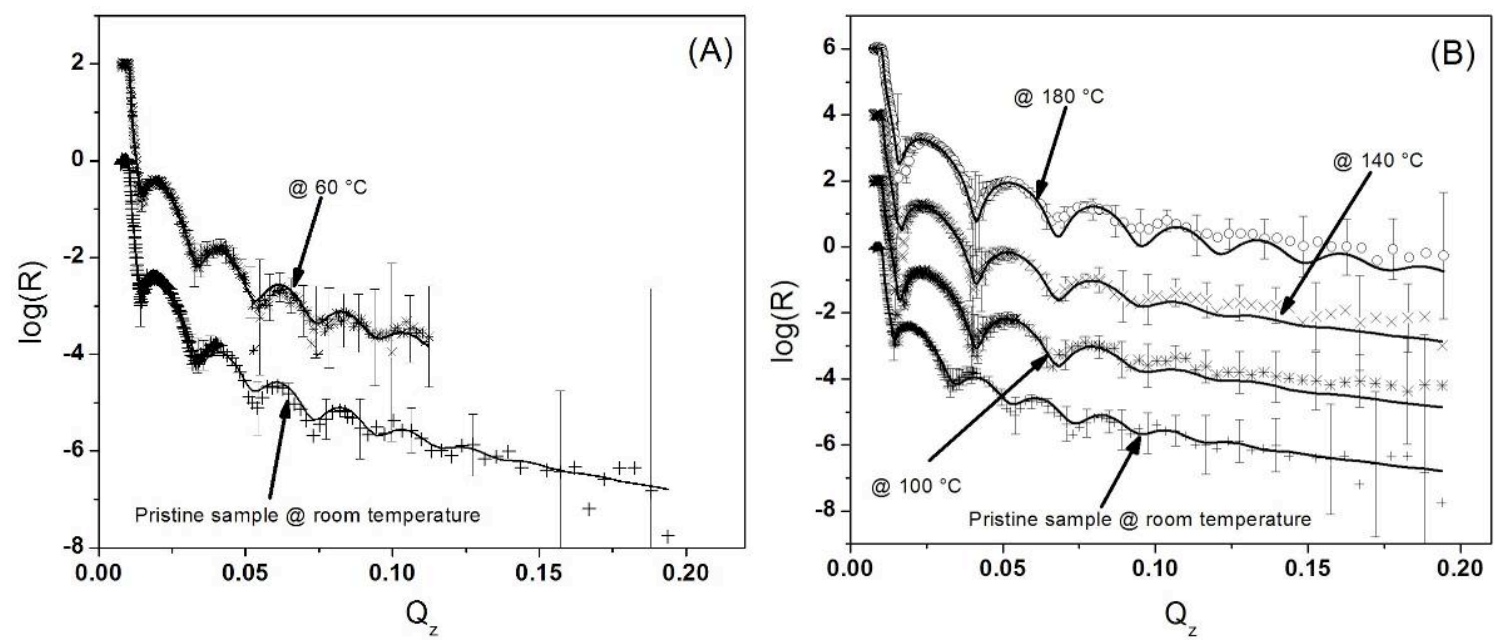

Figure 6. Raw neutron reflectometry data (symbols) for the $40 \mathrm{wt} \% \mathrm{AN}$ film, plotted as $\log$ reflectivity vs. $\mathrm{Q}_{\mathrm{z}}$. The data fit for each set of data is shown as a solid line. The data is split into two figures, (a) showing low temperature and (b) showing higher temperature, offset along y for clarity. In the data, fringe spacing (along $\mathrm{Q}_{\mathrm{z}}$ ) and height (along $\log (\mathrm{R})$ ) are determined by the SLD of the material and its thickness.

The SLD profile for each sample is given below in Figure 7, along with a comparison of all three samples at 60 and $100^{\circ} \mathrm{C}$. The summary data for the figures in tabular form is given in the Supplemental section, along with reference values from the literature. We note that the total film thickness from NR model fitting is within 3-4 $\mathrm{nm}$ of the ellipsometry data fitting for each sample, a good agreement. Each NR sample was modeled from bottom to top (left to right in the figure) as a silicon substrate with a native $\mathrm{SiO}_{2}$ layer coated by at least one sample layer in an air atmosphere.

This NR study provided a rich dataset from which several observations can be made. First, we compare SLD profile changes between samples. The SLD is not very different between the 
various materials and does not change much with temperature. As temperature increases, the SLD for AN would be expected to decrease across each possible phase transition. We see no evidence of phase transitions here. The SLD for the $25 \%$ and $40 \%$ AN samples are approximately equal but greater than for $10 \%$, as expected (AN SLD is greater than PVP SLD). All samples exhibit a small change in thickness and corresponding increase in density between 60 and $100^{\circ} \mathrm{C}$. This may be due to some retained solvent, despite drying efforts, high surface area to volume ratio samples, and waiting at least 48 hours between sample fabrication and NR measurement. It is possible that some methanol (negative SLD) is tightly bound to the AN, to the PVP, or between AN and PVP molecules. This effect is most dramatic in the $40 \%$ sample, suggesting an AN-based mechanism rather than PVP-based. Whatever the mechanism, it is observed to some extent in each sample, as highlighted in Figure $7 \mathrm{~d}$.

Second, we examine the evolution of SLD profile for a given sample. Each sample contains twophase structures in the AN-PVP film. We discovered this by attempting to fit the data using only one layer for the film (along with appropriate layers for the $\mathrm{Si}$ substrate and $\mathrm{SiO}_{2}$ native oxide) but the error for the fit was quite high. This was less apparent in the $10 \%$ sample but very apparent for the $40 \%$ sample. Each sample exhibits a slightly denser phase near the substrate and a less dense phase near the air-film surface. Because this effect increases with AN content, this indicates the AN is preferentially located near the silicon surface rather than the air surface. Many crystallization studies find similar results. In all samples, the two phase structure becomes less dramatic as temperature increases. This could add further credence to the earlier hypothesis of retained solvent, where the solvent would evaporate from the surface during heating. 
Significantly, if AN was decomposing and evaporating from the surface, the two-phase structure would become more dramatic with temperature.

We did observe an unusual feature in the $10 \mathrm{wt} . \%$ sample, wherein at $180^{\circ} \mathrm{C}$ the sample increased in thickness and had a significant decrease in overall SLD and two-phase structure. This temperature is slightly above the $T_{g}$ observed for the bulk and may give rise to foaming or other decomposition processes in the film sample. Whatever the process, it results in an SLD of essentially zero (presumably air) in the bulk of the film but some higher SLD material at the surface. This feature is not observed in the other samples. We further investigated this curiosity with PLM.

Figure 8 shows polarized light micrographs of the $10 \mathrm{wt} \%$ and $40 \mathrm{wt} \%$ samples after the heated neutron reflectometry experiments. The $10 \mathrm{wt} \%$ sample shows significant blister / delamination sites. They do not appear to be from small crystallites which individually evaporated but instead are relatively large. This indicates that substantial sections of the film raised up from the surface during the heating, which would explain the unusual SLD profile. It is unknown if the material did indeed foam or if some process (e.g. delamination or peeling of the film) was occurring during the heating. Further investigation is beyond the scope of this research but off-line heating experiments could reveal if this was a repeatable event. For the purposes of AN phase stabilization, film behavior above $150^{\circ} \mathrm{C}$ is not relevant. 

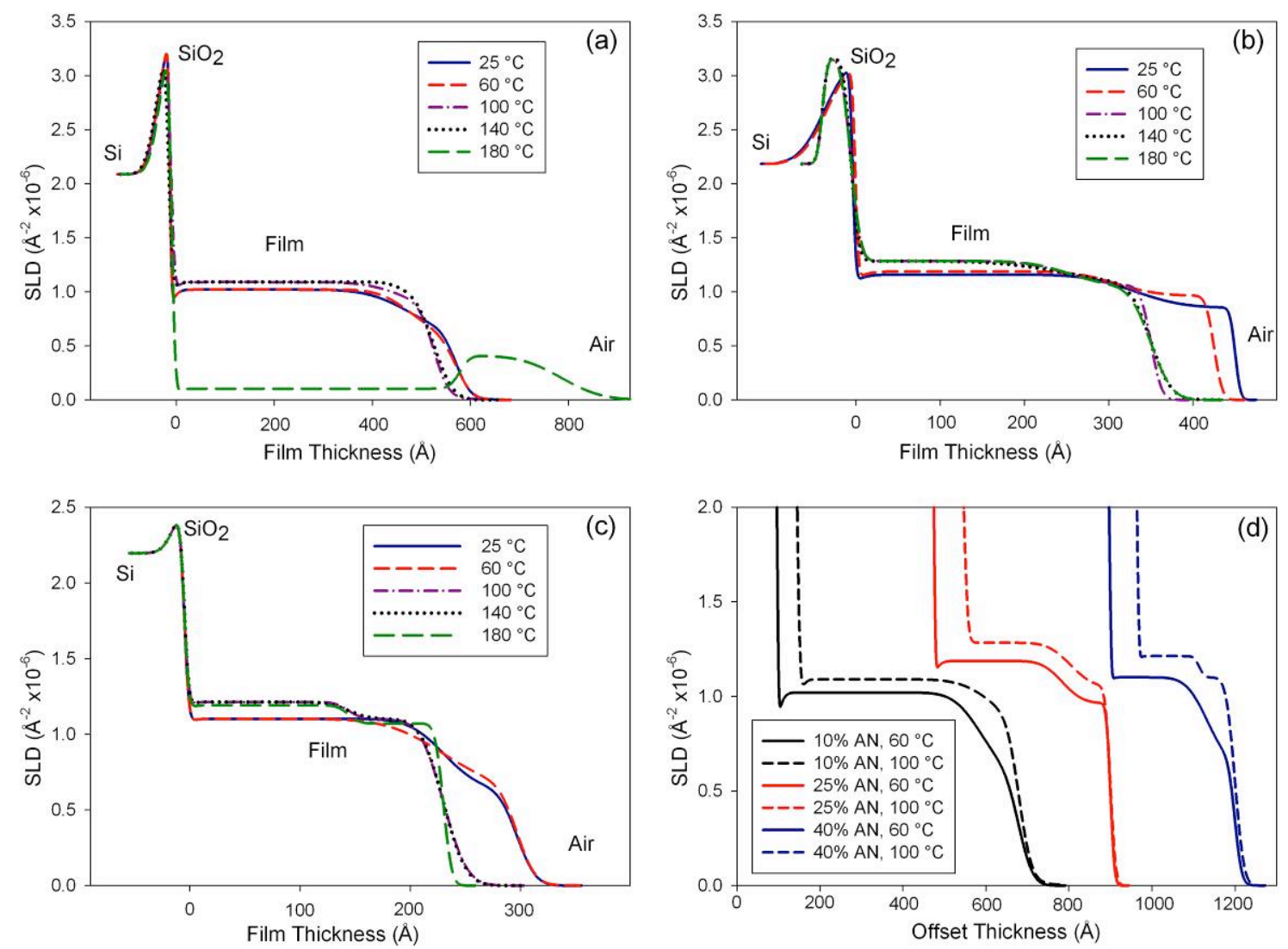

Figure 7. SLD profiles for the film samples as a function of temperature. "0" film thickness is the nominal substrate $\left(\mathrm{SiO}_{2}\right)$ surface. All temperatures are shown for the $10 \mathrm{wt} \%$ AN-PVP sample in (a), for the $25 \mathrm{wt} \%$ sample in (b), and the $40 \%$ sample in (c). All three samples are compared at 60 and $100^{\circ} \mathrm{C}$ in (d). 


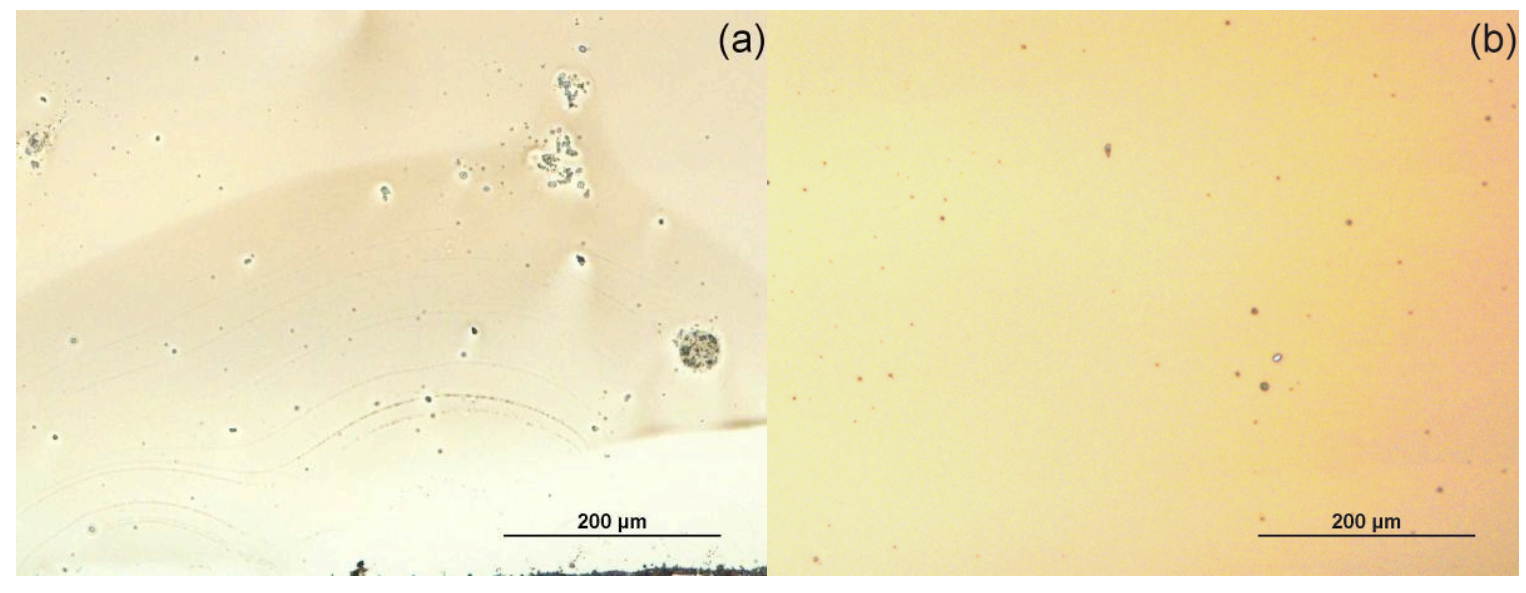

Figure 8. Polarized light micrographs of the $10 \mathrm{wt} \%$ sample (a) and $40 \mathrm{wt} \%$ sample (b). Extensive damage can be seen in (a) as evidenced by large variation in brightness across the film, indicating blisters or other delamination. Pockmark spots appear in both films and could be simply a PVP binder damage mechanism.

\subsection{Implications for AN Stabilization}

The results from the combination of techniques applied here give rise to several important conclusions regarding the suitability of PVP films for AN phase stabilization. To summarize the findings for AN loading: as AN content increased, crystallization became more likely (though still rare in the thin film) and the structure of the polymer increasingly became disrupted. Ellipsometry showed increasing deviation from the normal PVP refractive index with AN content. NR showed increasing two-phase structure in the sample, along with more dramatic densification of the sample between 60 and $100^{\circ} \mathrm{C}$. DSC-TGA, while conducted on bulk samples rather than films, indicated a stronger effect on $T_{g}$, where the $T_{g}$ response became more exothermic and happened over a narrower range of temperatures (more likely a time effect). As temperature increased, an initial densification of each film was observed, which is opposite of expected observation if the AN had undergone a phase transformation. Also, the two-phase 
structure in the film became less dramatic, indicating retained solvent or some similar mechanism for raising the SLD near the surface. Finally, an unusual structure was observed in the $10 \mathrm{wt} \%$ sample at $180^{\circ} \mathrm{C}$ which was caused by blisters forming from foaming or other decomposition processes.

Overall, we found no evidence of phase transformation even in the highly loaded films. The 10 wt $\%$ and $25 \mathrm{wt} \%$ films had a completely stable solid solution, while the $40 \mathrm{wt} \%$ film only showed significant AN precipitation in relatively thick regions (estimated $>100 \mathrm{~nm}$ ). This represents a substantial improvement over the work of Lang and Vyazovkin using bulk samples, where $25 \mathrm{wt} \%$ appeared to be the maximum concentration of AN in PVP. Here, if the composite film was sufficiently thin, very few AN crystallites were found. There are likely several factors involved in these samples. The films may be so thin that a critical nucleation volume for the AN is either unreachable (thermodynamic explanation) or only reachable at long times due to mass transport and diffusion issues (kinetic explanation). The kinetic explanation is more likely because critical nucleation in most materials occurs at less than $10 \mathrm{~nm}$, while our films were greater than $40 \mathrm{~nm}$. If it is a kinetically hindered process, the films are at worst metastable because crystallization did not occur in the region of interest even after several months. In any case, the high surface area of the films is not assisting phase transformation, as might be expected for environmentally-driven processes. A related hypothesis is that the extensive contact between the silicon substrate and the film plays a stabilizing role. Another consideration is that the retained solvent could be playing a role at ambient conditions. The AN may be somewhat stabilized by the interplay between AN, the PVP, and methanol. This explanation would be an extension of the scenario proposed by Lang and Vyazovkin. Identifying the underlying 
mechanism(s) for our PSAN is beyond our current scope but will certainly be the subject of further investigations, particularly in efforts to load even more AN into a polymer matrix.

This research suggests exciting opportunities for stabilizing AN or other oxidizing or explosive crystals in polymer binders. By taking advantage of the unique surface to volume ratios of extremely thin films, future research may be able to use innovative manufacturing methods to stabilize supersaturated films and assemble them into larger samples. We note that PVP is a useful candidate binder due to its availability and extensive characterization history, but there is no reason to exclusively study explosive-PVP mixtures. Indeed, explosive and propellant formulations have used many binders, ranging from the widely available to the highly specialized. Generally speaking, propellants use greater than $50 \mathrm{wt} \%$ loading of energetic materials, so increasing the loading of our PSAN films could result in wider applications to the propellants and explosives industries. Our future research efforts will also investigate the thermodynamic and kinetic interactions between crystals and binders to discover relationships between processing, structure and properties of the composite films.

\section{Conclusions}

Our research discovered that AN could be phase-stabilized in PVP films at higher loading than previously reported for bulk samples. Investigating this effect in detail, ellipsometry and PLM revealed that high loading of AN results in solid-solution heterogeneity in a stable glassy ANPVP film, but at greater thicknesses this heterogeneity can result in crystallization of the AN. This suggests that there is some critical film thickness below which the AN segregates or accumulates to some extent without crystallizing. We see no evidence of the multiple solid-solid 
phase transitions known to occur in $\mathrm{AN}$ between 50 and $150^{\circ} \mathrm{C}$, presumably because the $\mathrm{AN}$ is kept in a glassy or intermixed phase and therefore does not behave like a crystal or powder. All films do have evidence of retained methanol solvent from the fabrication procedure, despite long soak periods at elevated temperature. This effect is more pronounced at higher AN concentrations, indicating some binding of the retained methanol to the AN. After the solvent evaporates between 60 and $100^{\circ} \mathrm{C}$, the remaining film still has a lower physical density than expected. In future research efforts, the solvent will be altered and the ammonium nitrate loading will be more significantly varied in order to study this effect. However, we clearly demonstrate that the interactions between the crystal and the binder are beneficial for phase stabilization, even more dramatically in the film than in the bulk, and it may be possible to stabilize greater than $50 \%$ AN by weight in a film system.

\section{Acknowledgements}

The authors wish to thank D. Dattelbaum (LANL) for helpful discussion and for provision of material. Los Alamos National Laboratory, an affirmative action equal opportunity employer, is operated by Los Alamos National Security, LLC, for the National Nuclear Security Administration of the U.S. Department of Energy under contract DE-AC52-06NA25396. This work was performed, in part, at the Lujan Neutron Scattering Center at LANSCE funded by the

DOE Office of Basic Energy Sciences. Funding for this work was provided by the Explosives Science project of the National Nuclear Security Administration Science Campaign 2.

\section{References}

1. Weston CW, Papcun JR, and Dery M. Ammonium Compounds. Kirk-Othmer Encyclopedia of Chemical Technology, vol. 3: John Wiley \& Sons, Inc., 2000. pp. 263-287.

2. Oommen C and Jain SR. Journal of Hazardous Materials 1999;67(3):253-281. 
3. Chellappa RS, Dattelbaum DM, Velisavljevic N, and Sheffield S. The Journal of Chemical Physics 2012;137(6):-.

4. Klimova I, Kaljuvee T, Türn L, Bender V, Trikkel A, and Kuusik R. Journal of thermal analysis and calorimetry 2011;105(1):13-26.

5. Kajiyama K, Izato $\mathrm{Y}-\mathrm{i}$, and Miyake A. Journal of thermal analysis and calorimetry 2013;113(3):1475-1480.

6. Tan L, Wu Q, Chen X, Jiang W, Xu S, and Liu D. Journal of Thermal Analysis and Calorimetry 2014;115(2):1759-1766.

7. Vargeese AA, Mija S, and Muralidharan K. Journal of Energetic Materials 2014;32(3):146-161.

8. Lang AJ and Vyazovkin S. The Journal of Physical Chemistry B 2008;112(36):11236-11243.

9. Jablonski AE, Lang AJ, and Vyazovkin S. Thermochimica Acta 2008;474(1):78-80.

10. Lang AJ and Vyazovkin S. Materials Letters 2008;62(12):1757-1760.

11. Wu H-D, Wu I-D, and Chang F-C. Polymer 2001;42(2):555-562.

12. Kestilä E and Valkonen J. Thermochimica Acta 1994;233(2):219-222.

13. Ingman JS, Kearley GJ, and Kettle SF. Journal of the Chemical Society, Faraday Transactions 1: Physical Chemistry in Condensed Phases 1982;78(6):1817-1826.

14. Wei Y and Cai BH. Advanced Materials Research 2012;399:1989-1993.

15. Rudjak I, Kaljuvee T, Trikkel A, and Mikli V. Journal of thermal analysis and calorimetry 2010;99(3):749-754.

16. Oxley JC, Smith JL, Rogers E, and Yu M. Thermochimica acta 2002;384(1):23-45.

17. Oxley JC, Kaushik SM, and Gilson NS. Thermochimica Acta 1989;153:269-286.

18. Yeager JD, Dubey M, Wolverton MJ, Jablin MS, Majewski J, Bahr DF, and Hooks DE. Polymer 2011;52(17):3762-3768.

19. Zhong C and Chu CC. Crystal Growth \& Design 2010;10(12):5043-5049.

20. Yeager JD, Ramos KJ, Mack NH, Wang H-L, and Hooks DE. Crystal Growth \& Design 2012;12(11):5513-5520.

21. Marentette JM and Brown GR. Polymer 1998;39(6-7):1415-1427.

22. Majewski J, Kuhl TL, Wong JY, and Smith GS. Reviews in Molecular Biotechnology 2000;74(3):207-231.

23. Yeager JD, Ramos KJ, Sun CC, Singh S, Dubey M, Majewski J, and Hooks DE. Molecular Pharmaceutics 2012;9(7):1953-1961.

24. Dubey $M$, Jablin $M$, Wang $P$, Mocko $M$, and Majewski J. The European Physical Journal Plus 2011;126(11):1-11.

25. Hickmott D, Lerner AH, Wang P, Majewski J, and Taylor M. Neutron Reflectometry at Elevated Pressures and Temperatures - Novel P-T Cell and Preliminary Experiments. American Geophysical Union Fall Meeting 2011, 2011.

26. Theeten JB and Aspnes DE. Annual Review of Materials Science 1981;11(1):97-122.

27. Nelson A. Journal of Applied Crystallography 2006;39(2):273-276.

28. Kahle O, Wielsch U, Metzner H, Bauer J, Uhlig C, and Zawatzki C. Thin Solid Films 1998;313314(0):803-807. 
